\title{
Ethics education and emotions
}

\section{Henk ten Have ${ }^{1}$}

Published online: 16 March 2020

(C) Springer Nature Switzerland AG 2020

In his dialogue Phaedrus Plato examines the nature of the soul. This is important to clarify the pursuit of philosophy as the true culture of the soul in its quest for truth, beauty and goodness. Here Plato introduces the image of the tripartite soul: a charioteer and two horses. The task of the charioteer which represents reason is difficult since it has to govern the horses of desire and emotion. In this allegory, the reflective part of the soul controls its passionate and appetitive parts. Emotion or passion is represented by the black horse. It needs to be subjugated by reason (Hackforth 1972). Although Plato with this image intended to clarify that reason cannot be divorced from desire and passion, the effect of the image has been that emotions were located outside of the rational component of the soul as irrational drives. To accomplish the ideal of wisdom, philosophers should detach from passions and desires emerging from the mortal body. Emotions are regarded as interfering with rational thinking.

This traditional view is reflected in ethics. Ethics is moral reasoning. It proceeds from rules and principles that have a rational basis, and that provide arguments for moral judgments and actions. Ethics also articulates impartiality and universality; rational deliberation and argumentation resulting in choices and decisions that can be justified. This is also the common assumption of bioethics: it is rational debate based on scientific evidence and facts; moral judgments are based on logical reasoning and ethical principles. There is no place for emotions and feelings. However, this view of moral agency is nowadays increasingly contested. Reason and emotion are intimately connected in ethics. As a particular branch of philosophy, ethics proceeds from moral experience. Human beings understand themselves and/in the world in moral terms. Ethics is the interpretation and explanation of that primordial understanding. Before practicing ethics we already know, at least to some extent, what is morally desirable, obliged or right. Otherwise, we would not recognize what is appealing in a moral sense. But on the other hand, what we recognize in our experience is unclear and in need of elucidation and interpretation. We approach the moral dimension of the world from a set of prior understandings; they form the basis of our interest in what seems strange and bizarre to us, and what makes us reconstruct its meaning(s). We should, therefore,

Henk ten Have

tenhaveh@duq.edu

1 Center for Healthcare Ethics, Duquesne University, Pittsburgh, PA, USA 
reflect upon our own moral experience as well as upon the moral sources and preunderstandings of our culture and tradition. Moral experience involves primarily feelings, for instance, of indignation, confusion or contentment; secondarily, these emotional responses can be made the object of moral thinking. A sharing of moral experiences of patients and physicians, and of the emotions and attitudes involved, is therefore required. Understanding and defining the morally relevant facts of a case do not merely involve the identification of relevant general principles and the deduction of a set of rules from which the correct response to the problem can be derived. The role of bioethics is not so much to explicate and apply ethical theories and principles but to interpret and evoke what is implied in moral experience. Another aspect is that before moral argument and judgment can be made, there is a preceding phase of moral perception in which situations are perceived as morally significant and relevant (Blum 1994) Ethical discourse recognizes that a gap exists between particular situations and moral rules and principles, which is bridged by moral judgment. But it does not take into account that before a moral judgment is delivered, moral perception is needed. This involves moral sensibility before a moral judgment can be made and rules and principles can be applied. Such sensibility emerges from understanding the particularity of the situation but also from recognizing particular moral features such as discomfort, distress, and compassion. If someone is not sensitive to the moral features of situations, he may not perceive them. Recognizing the significance of situations as morally relevant calls for moral perception of injustice, distress, dishonesty, and indignity. Such perception is promoted by the imagination, expanding our sympathies and imaging ourselves in the situation of other people. Before moral actions are taken, there are, what Blum calls, 'nonactional responses,' elicited by the imagination and invoking emotions (Blum 1994, p.57). That moral reasoning often is not the outcome of previously formed judgments is famously argued by Greene and Haidt (2002). It is a mistake to assume that moral judgments are the result of moral reasoning. According to these scholars, it is many times the product of post-hoc rationalization of judgment. Moral actions are more related to moral emotions than to moral reasoning. The dominant rationalist model of moral judgment therefore is deficient. In fact, there are two cognitive processes at work: reasoning and intuition. Emotion and intuition are a kind of cognition (Haidt 2001).

In the context of healthcare, emotions are also regarded as irrational and subjective. Within the dominant materialistic and naturalistic world view of medical science, the soul and thus subjectivity are not especially relevant. Emotions and feelings such as empathy and compassion should not play a role in the proceedings of medical practice. The image of the competent professional is detached concern. Doctors should not become emotionally involved in caring for their patients. They should maintain some distance in order to maintain their objectivity. At the same time, it is acknowledged that interpersonal relations and empathic understanding are important. Doctors cannot merely be detached rational agents. But detachment is an important ethical value, and that is what they learn in medical school. The ideal is that they are neutral moral agents who do not allow emotions to interfere with their scientific approach.

Detachment is justified with several arguments. It is a way to protect oneself from burnout. Practitioners have to concentrate on painful procedures and they can only execute them when they are not too much involved in the patient's personal circumstances. It is also necessary to care for patients in an impartial way. Professionals cannot 
let their emotions and feelings determine how they will act. Emotions are subjective and can easily interfere with objective and rational judgment. Professionals should be free from bias and prejudice.

Traditionally, sympathy and compassion have always been regarded as crucial for medical practice, and especially for contributing to healing. In medicine it is impossible to ignore the needs of patients for emotional interactions with physicians and other care providers. Attending to the subjective experiences of patients will not only deliver important information but will also engage physicians in more effective communication. It will build trust with patients and will improve the effectiveness of medical treatment. The concept of relational autonomy is helpful in this regard. It is essential for patient-centered care because it focuses attention to the patient as a whole person within his or her social context. It also enhances shared decision-making, communication and partnership, supporting the autonomous capacities of the patient. The image of detached concern therefore is unsatisfactory (Halpern 2010). Empathy should supplement objective knowledge. A long time ago, George Engel, in his critique of the biomedical model, denounces the model as cold and impersonal. It is not only unsatisfying for patients but also for physicians. If they are insensitive to the personal problems of patients and their families, they experience disenchantment and feel that they neglect their patients (Engel 1977). The negative effects of such disenchantment are increasingly recognized nowadays. A recent Editorial in The Lancet signals a global crisis of physician burnout. In the United States, $78 \%$ of physicians are affected by burnout; in the United Kingdom $80 \%$. Although exact data are not available, similar phenomena of emotional exhaustion, de-personalization, and a sense of reduced personal accomplishment are observed in many other countries. It is not only a tragedy for individual physicians but is also threatening healthcare systems and patients' care (Editorial 2019).

Feelings of negativism and cynicism are encouraged during medical education. It is a common finding since a long time that empathy is declining in medical school and residency. It is estimated that $75 \%$ of medical students become more cynical about the medical profession especially in the first 4 years of their education (Newton et al. 2008; Neumann et al. 2011). This education emphasizes emotional detachment, affective distance and clinical neutrality. Reasons for decline of empathy and idealism are not clear. It may be distress because of mistreatment by superiors and mentors, negative role models, failure to deal with anxieties concerning illness and suffering, vulnerability, social support problems, emphasis on financial regulations, and high workload. Anyway, the result is that students identify "with a cold and uncaring role model" (Hojat et al. 2004, p. 938) This is a reason for concern since empathy and compassionate care are generally regarded as essential for good relationships between patient and physician. The learning environment is usually blamed. There is an absence of specific educational programs to cultivate humanistic qualities. There is need for systematic training of such qualities in medical schools. But most probably more will be needed to nurture empathy.

What about emotions in ethics education? That it is an important issue is clear from many publications. There is a wealth of literature produced for example by medical students and residents about experiences in medical school and clinical training. Surveys but also diaries and autobiographies testify that students often face challenging emotional experiences (Kremer et al. 2019; Weurlander et al. 2019). 
But how is the role of emotions addressed in education? In this issue, Amalia Muhaimin and colleagues report a study comparing how clinical students in Indonesia and the Netherlands deal with emotional challenges in ethics (Muhaimin et al. 2020). They make a helpful distinction between three emotional settings in which ethical problems arise: feelings of not performing well enough, witnessing unethical or unprofessional behaviors of superiors and co-workers, and limitations of the working environment, especially bureaucracy and limited resources. In these settings, students may feel distressed and frustrated, and ethical reflection and self-evaluation particularly in group discussions, may help to deal with these emotions, and thus to clarify significant moral values involved. Muhaimin and colleagues therefore conclude that it is important to address the emotional experiences of students in ethics education.

We are looking forward to receive more contributions about this important dimension of ethics education. An excellent occasion to exchange experiences will be the forthcoming 8th International Conference on Ethics Education that will take place in Zagreb, Croatia, July 3-5, 2020. The conference will be organized by the International Association for Education in Ethics (IAEE) with the Andrija Stampar School of Public Health, School of Medicine, University of Zagreb, the Croatian Bioethics Society, and the Croatian Centre of Excellence for Integrative Bioethics as the co-organizers. The core focus of the conference, titled Ethics Education, Interdisciplinarity and Pluriperspectivism, will be on bringing together different scientific and cultural perspectives in the field of ethics education. For more information, see: www. ethicsassociation.org; http://www.bioetika.hr/iaee2020/.

\section{References}

Blum, Lawrence A. 1994. Moral perception and particularity. New York: Cambridge University Press.

Editorial. 2019. Physician burnout: a global crisis. The Lancet 394 (10193): 93.

Engel, George L. 1977. The need for a new medical model: A challenge for biomedicine. Science 196 (4286): 134.

Greene, Joshua, and Jonathan Haidt. 2002. How (and where) does moral judgment work? Trends in Cognitive Sciences 6 (12): 517-523.

Hackforth, R. 1972. Plato's Phaedrus. Cambridge: Cambridge University Press.

Haidt, Jonathan. 2001. The emotional dog and its rational tail: A social intuitionist approach to moral judgment. Psychological Review 108: 814-834.

Halpern, Jodi. 2010. From detached concern to empathy. Humanizing medical practice. Oxford/New York: Oxford University Press.

Hojat, Mohammadreza, Salvatore Mangione, Thomas J. Nasca, Susan Rattner, James B. Erdmann, Joseph S. Gonnella, and Mike Magee. 2004. An empirical study of decline in empathy in medical school. Medical Education 38: 934-941.

Kremer, Telma, Sylvia Mamede, Milton A. Martins, Patricia Tempski, and Walter W. van den Broek. 2019. Investigating the impact of emotions on medical students' learning. Health Professions Education 5 (2): 111-119.

Muhaimin, Amalia, Maartje Hoogsteyns, Adi Utarini, and Derk Ludolf Willems. 2020. Ethics education should make room for emotions: a qualitative study of medical ethics teaching in Indonesia and the Netherlands. International Journal of Ethics Education 5 (1) this issue.

Neumann, Melanie, Friedrich Edelhäuser, Diethard Tauschel, Martin R. Fischer, Markus Wirtz, Christiane Woopen, Aviad Haramati, and Christian Scheffer. 2011. Empathy decline and its reasons: A systematic review of studies with medical students and residents. Academic Medicine 86 (8): 996-1009.

Newton, Bruce W., Laurie Barber, James Clardy, Elton Cleveland, and Patricia O'Sullivan. 2008. Is there a hardening of the heart during medical school? Academic Medicine 83 (3): 244-249. 
Weurlander, Maria, Annelena Lönn, Astrid Seeberger, Hakan Hult, Robert Thornberg, and Annika Wernerson. 2019. Emotional challenges of medical students generate feelings of uncertainty. Medical Education 53 (10): 1037-1048.

Publisher's note Springer Nature remains neutral with regard to jurisdictional claims in published maps and institutional affiliations. 